\title{
Control System for Auto Moving Vehicle using for Artificial Turf Ground Performance Test
}

\author{
M.J. Chung
}

\author{
Myung Jin Chung* \\ Mechatronics Engineering Department \\ Korea Polytechnic University, Republic of Korea \\ *Corresponding author: mjchung@kpu.ac.kr
}

\begin{abstract}
Recently, use of artificial turf has been increasing in a variety of international games as well as the change of school playgrounds to artificial turf from clay. Artificial turf is needs to obtain a standard certification to hold international games. This standard certification is based on a shock performance test during a specified period. The control system of the auto moving vehicle using for artificial turf ground performance tests is proposed and the control algorithm was adopted to improve the trajectory following performance of the auto moving vehicle. The control algorithm was optimized by computer simulation. Each part of the control system was developed and integrated into the auto moving vehicle. Experiments were conducted to verify the performance of the control system. From the performance test, the control system had a positioning resolution of $0.05 \mathrm{~m}$, maximum velocity of $1 \mathrm{~m} / \mathrm{sec}$, and a trajectory following error of $\pm 1.3 \mathrm{deg}$ for operating conditions.

Keywords: control system, auto moving vehicle, artificial turf, positioning, velocity, trajectory following.
\end{abstract}

\section{Introduction}

The use of artificial turf has been increasing from the variety of international games held on it and the change of school playgrounds to artificial turf from clay. Artificial turf ground is required to meet the standards of certification to play international games. This standard certification is given by the Federation International Football Association (FIFA) rules determined by a shock performance test during a specified period. FIFA rules are described in the "FIFA Quality Concept - Handbook of Test Methods and Requirements for Artificial Turf Football Surfaces." Shock performance test technology is growing due to the development of automobile crash safety performance test technology. However, the application area of shock performance test technology is limited to the automobile industry. [1] Data acquisition and analysis technologies are limited to the automobile industry as well. Currently, the shock performance test for artificial turf ground is conducted by manually moving the test system around the testing area of the artificial turf. It is a time consuming process and the repeatability of the measured data is poor. [2] Therefore, an auto measuring system having functions including auto moving, auto testing, and auto data acquisition and analysis is required for the shock performance test.

Researches associated with auto moving vehicles and auto shock performance tests are conducted in the area of automobile crash safety performance tests, respectively. [3]- [5] Researches on the combined control of auto moving vehicles and auto shock performance test are rarely conducted in the area of artificial turf. [6]- [7] In addition, improvement of the trajectory following performance of the auto moving vehicle is required for application in the auto measuring system for the shock absorbing performance test of artificial turf.

In this work, the control system of the auto moving vehicle using for the artificial turf ground performance test is proposed and the control algorithm was adopted to improve the trajectory following performance of the auto moving vehicle. The control algorithm was optimized by computer simulation. Each part of the control system was developed and integrated into the 
auto moving vehicle. Experiments were conducted to verify the performance of the developed control system of the auto moving vehicle.

\section{Configuration}

The configuration of auto measuring system for shock absorbing performance test of artificial turf ground is as follows: auto moving vehicle for moving the auto shock performance testing module, auto shock performance testing module for raising and dropping the reference weight, data acquisition module for measuring the force and acceleration of the reference weight, and wireless communication module for transferring the measured data from the data acquisition module to the remotely positioned PC as shown in Fig. 1. The auto moving vehicle is operated as described in Fig. 2. The auto moving vehicle containing the auto shock performance testing module follows the reference trajectory of the artificial turf ground, and stops for shock performance testing. When the auto moving vehicle stops, the auto shock performance testing module raises and drops the reference weight. Then, the data acquisition module measures the force and acceleration of the reference weight and the wireless communication module transfers the measured data from the data acquisition module to the remotely positioned PC. In the remote $\mathrm{PC}$, measured data is analyzed and graphically displayed. The control system of the auto moving vehicle consists of two BLDC hub motors and drivers, six Ni batteries, digital magnetic compass sensor, ZigBee transmitter and receiver, and a main controller as shown in Fig. 3.

\section{Requirements}

The requirements of the auto measuring system for the shock absorbing performance test of artificial turf include the auto vehicle moving along the reference trajectory of the artificial turf ground, auto shock performance testing at the reference position, auto data acquisition of measured data, and the auto transferring of the measured data to the remotely positioned PC via wireless communication. The requirements of the auto moving vehicle also include a positioning resolution of $0.05 \mathrm{~m}$, maximum velocity of $1 \mathrm{~m} / \mathrm{sec}$, and a trajectory following error of $\pm 2.0 \mathrm{deg}$ along the reference trajectory of the artificial turf with an area of $100 \mathrm{~m}$ by $200 \mathrm{~m}$. The control system of auto moving vehicle is required to have a robust trajectory following performance.

\section{Design and Development}

The control system of the auto moving vehicle consists of two BLDC hub motors and drivers, six Ni batteries, digital magnetic compass sensor, ZigBee transmitter and receiver, and a main controller. Two 250watt BLDC hub motors and two BLDC motor drivers are used for moving the $40 \mathrm{~kg}$ auto shock performance testing module as shown in Fig. 4. The specifications of the BLDC hub motor and driver are listed in Table 1. Six Ni batteries with capacities of $40 A H$ are connected in parallel. A digital magnetic compass sensor with a $0.5 \%$ resolution is used to detect the trajectory following direction of the auto moving vehicle as shown in Fig. 5. The main controller uses a 16bit microprocessor for signal processing and control algorithm as shown in Fig. 5. In the main controller, by comparing the reference direction signal and the digital magnetic compass, the motor driving sensor signal is generated, fed to the BLDC motor driver, and used to control its velocity and position. The position data is calculated with the digital magnetic compass sensor signal and the BLDC motor hall sensor signal. The wireless communication module consists of the ZigBee transmitter and receiver with a real-time transferring speed of $115 \mathrm{Kbps}$ and a transferring distance of $1 \mathrm{~km}$ as shown in Fig. 6. This module receives the 


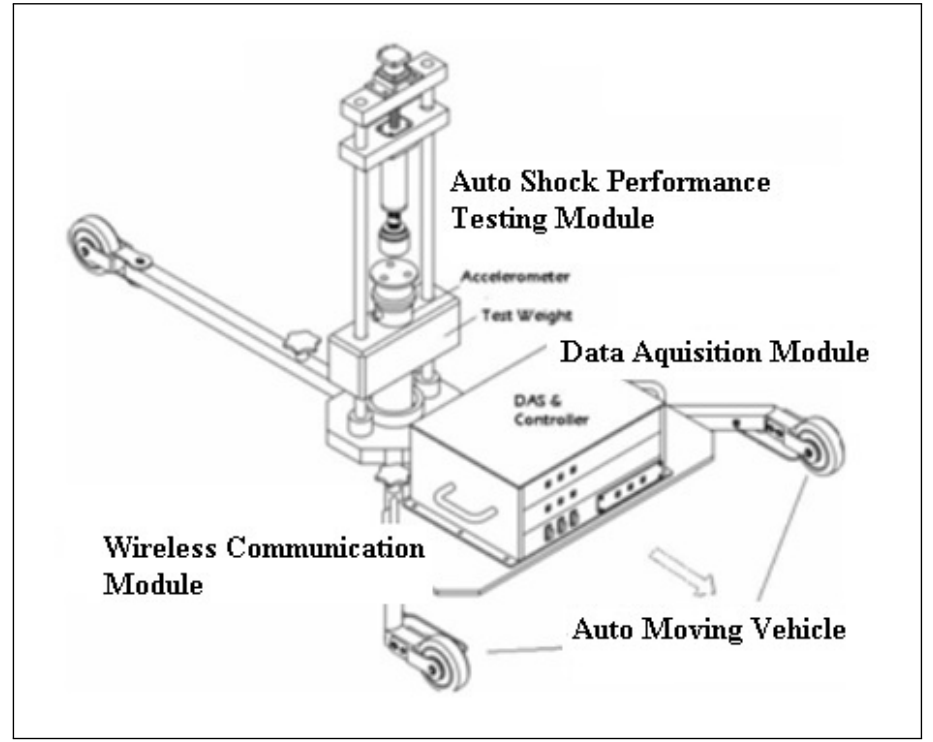

Figure 1: Configuration of auto measuring system

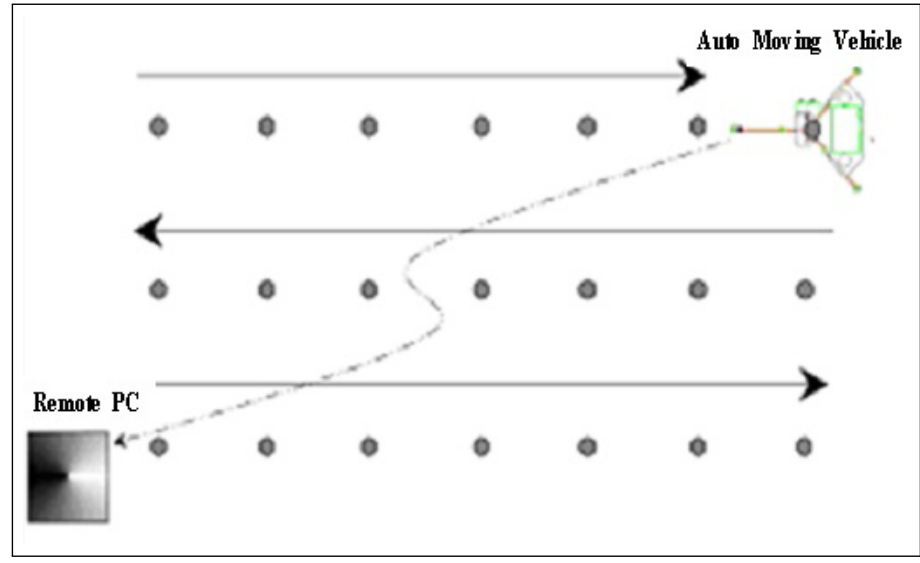

Figure 2: Operation procedure of auto moving vehicle

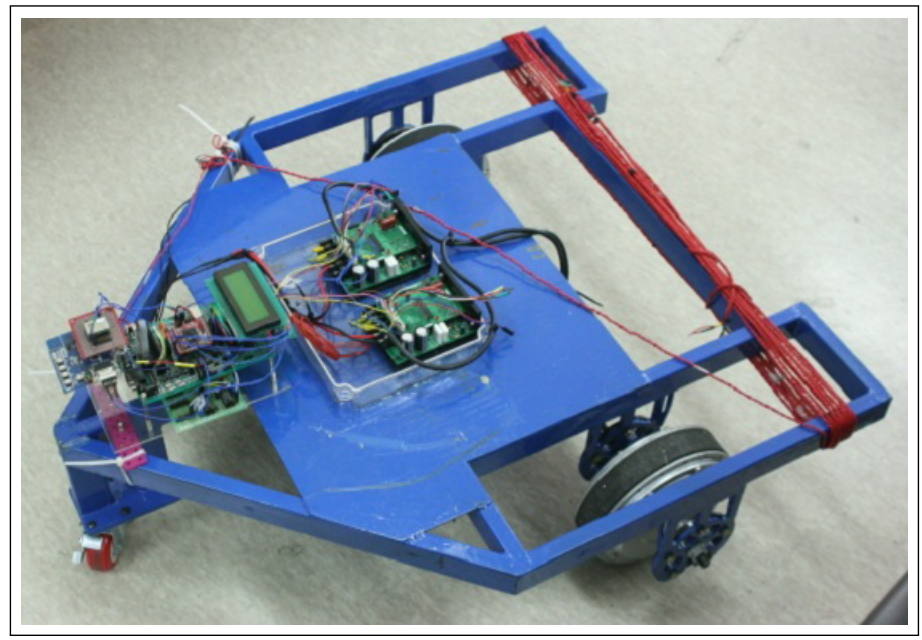

Figure 3: Control system of auto moving vehicle 
operating signal from the remote $\mathrm{PC}$ and transmits the measured data to the remote $\mathrm{PC}$ for analysis.

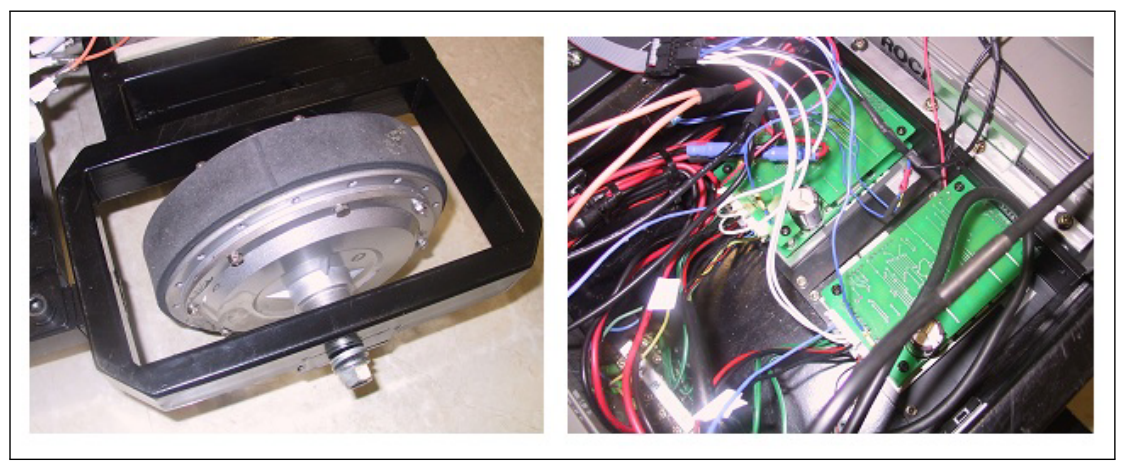

Figure 4: BLDC hub motor and driver

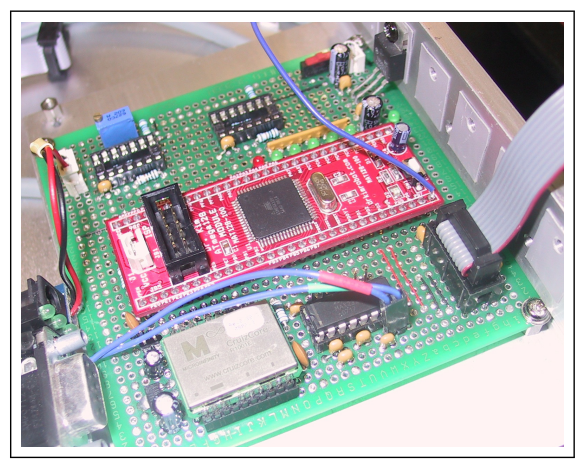

Figure 5: Compass sensor and main controller

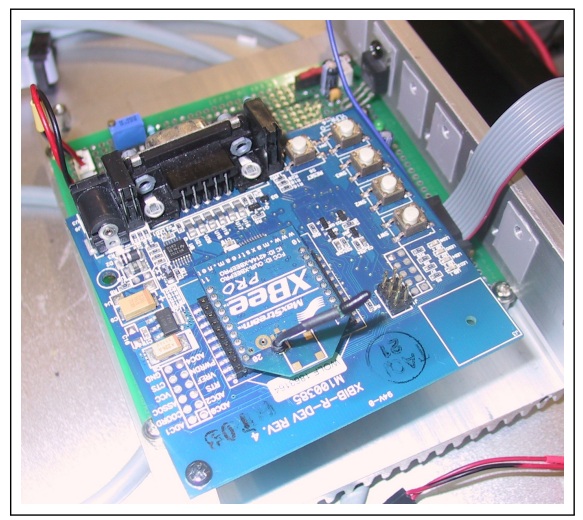

Figure 6: Wireless communication module

\section{Control Algorithm}

The control algorithm was adopted to improve the trajectory following performance of the auto moving vehicle and was optimized by using a computer simulation. The auto moving vehicle 
Table 1: Specifications of the BLDC hub motor and driver

\begin{tabular}{|c|l|c|c|}
\hline & Parameters & Value & Unit \\
\hline Motor & Power & 250 & Watt \\
& Input voltage & 36 & Volt \\
& Rated RPM & 3000 & RPM \\
& Hub wheel size & 200 & $\mathrm{~mm}$ \\
\hline Driver & Rated voltage & 48 & Volt \\
& Rated current & 10 & $\mathrm{~A}$ \\
& Velocity control method & $\mathrm{PWM}$ & - \\
& Switching Frequency & 10 & $\mathrm{kHz}$ \\
\hline
\end{tabular}

can be described by the mathematical modeling of the motor and body of the auto measuring system. Electric model of the motor is given by:

$$
\begin{gathered}
V-R i-L \frac{d i}{d t}-K_{b} \dot{\theta}=0 \\
T_{w}=K_{T} i
\end{gathered}
$$

where $V$ is a voltage applied to the motor, $R$ is a resistance of the motor, $i$ is a current flowing through the motor, $L$ is an inductance of the motor, $K_{b}$ is a constant of back EMF, $T_{w}$ is the torque generated by the motor, and $K_{T}$ is the constant of torque, respectively.

Dynamic model of the motor and body of auto measuring system is given by: [8]

$$
\begin{aligned}
2\left(m r^{2}+I_{w}\right) \ddot{\theta} & =2 T_{w}+R_{H} r \\
M r^{2} \ddot{\theta} & =R_{H} r
\end{aligned}
$$

where $m$ is a mass, $r$ is a radius, and $I_{w}$ is the moment of inertia of the motor, $M$ is the mass of the body, and $R_{H}$ is the horizontal force acting on the motor by the body of the auto measuring system, respctively.

From Eq. (1) - (4), the relationship of angular velocity $(\Omega)$ and applied voltage $(V)$ is geiven by:

$$
\begin{gathered}
T_{w}(s)=\frac{K_{T}}{L s+R}\left[V(s)-K_{b} \Omega(s)\right] \\
\frac{\Omega(s)}{T_{w}(s)}=\frac{2}{\left.\left[(2 m+M) r^{2}+2 I_{w}\right)\right] s}
\end{gathered}
$$

From Eq. (5) and (6), simulink block diagram of the control system of the auto moving vehicle can be constructed as shown in Fig. 7. In this block diagram, a PID controller was adopted to optimize the trajectory following performance of the auto moving vehicle. The control signal generated by the PID controller is given by:

$$
u(t)=K_{p} e(t)+K_{i} \int_{0}^{t} e(\tau) d \tau+K_{d} \frac{d e(t)}{d t}
$$

where $\mathrm{u}$ is a control signal, $K_{p}$ is a proportional, $K_{i}$ is a integral, and $K_{d}$ is a derivative coefficient, respectively. In this system, $K_{i}$ value is sensitive to the deviation range of the directional angle. 


\section{$6 \quad$ Experimental Results}

The auto measuring system including the developed control system of the auto moving vehicle was used as an experimental setup for performance verification as shown in Fig. 8. In this experimental setup, the angle of the digital magnetic compass sensor was set at 0 degrees at the starting point and the signal processing time was set at $50 \mathrm{msec}$. Fig. 9 shows the experimental results of the auto moving vehicle according to the control signal for a $40 \mathrm{~m}$ straight line following motion. In this experiment, the positioning resolution was $0.05 \mathrm{~m}$, maximum velocity was $1 \mathrm{~m} / \mathrm{sec}$, and the deviation range of the directional angle was $\pm 1.3 \mathrm{deg}$, respectively.

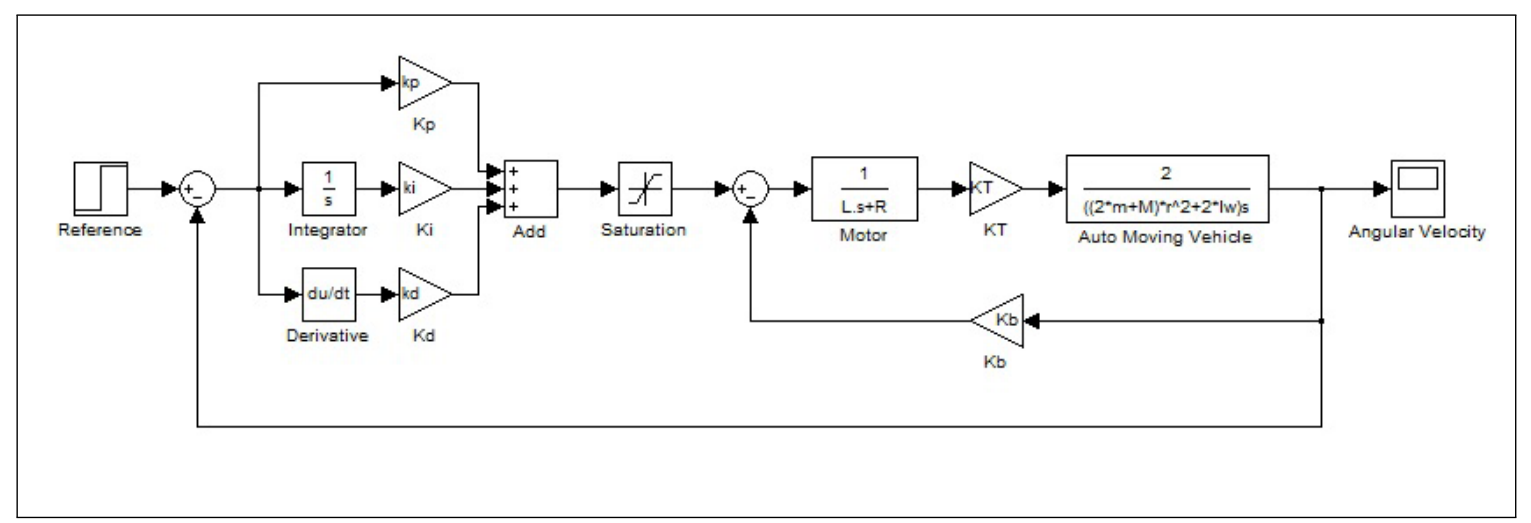

Figure 7: Simulink block diagram for PID controller simulation

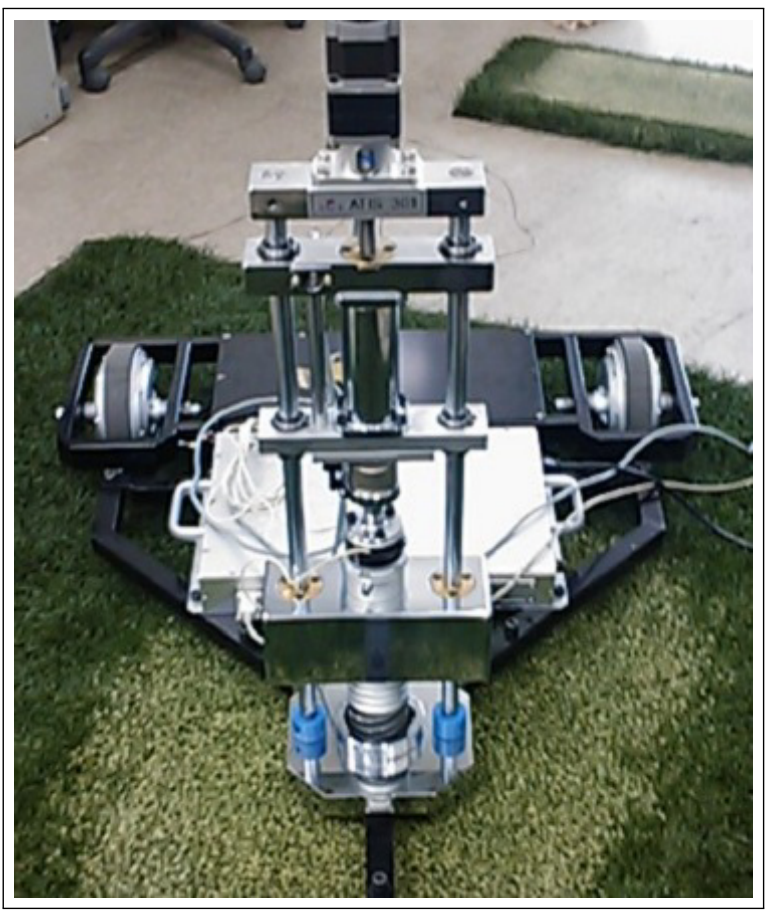

Figure 8: Experimental setup of the developed auto moving vehicle 


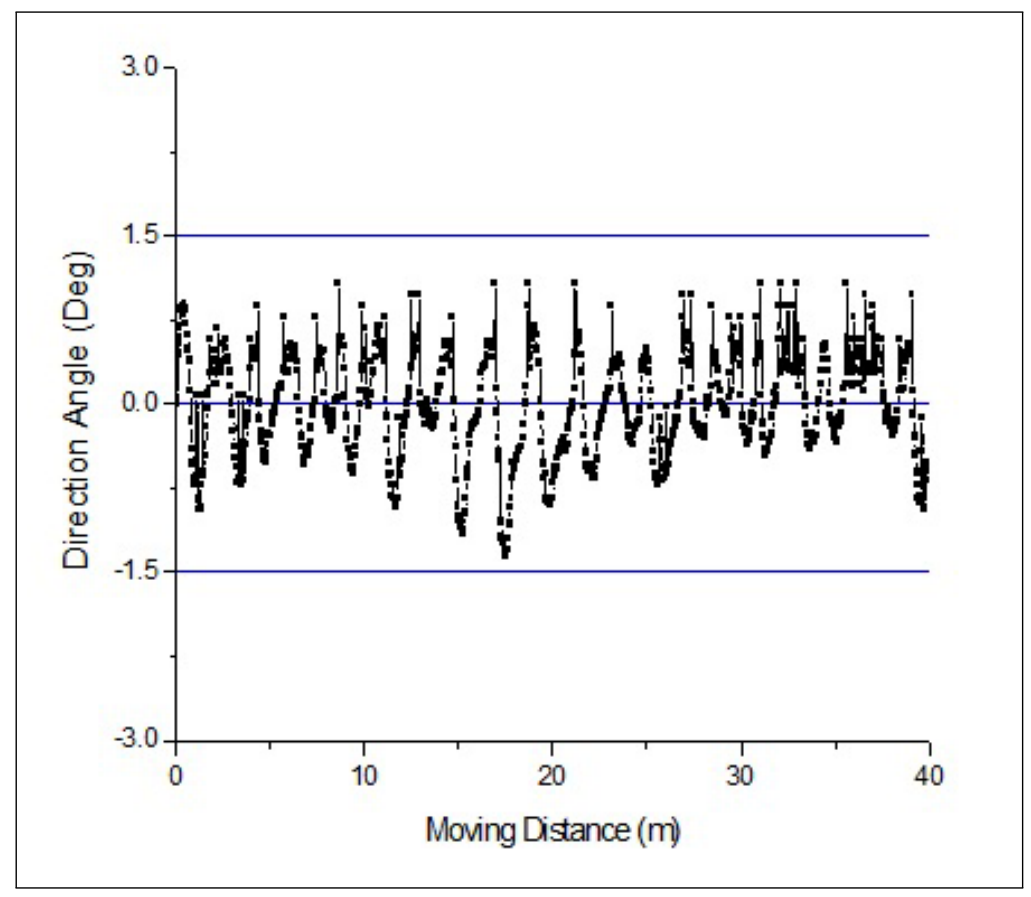

Figure 9: Experimental result of the auto moving vehicle

\section{Conclusions}

From this work, the control system of the auto moving vehicle using for artificial turf performance tests was proposed and a control algorithm to improve the trajectory following performance of the auto moving vehicle was adopted. The control algorithm was optimized by computer simulation. Each part of the control system was developed and integrated into the auto moving vehicle. Experiments were conducted to verify the performance of the developed control system of the auto moving vehicle. From the performance test, the developed control system of the auto moving vehicle had a positioning resolution of $0.05 \mathrm{~m}$, maximum velocity of $1 \mathrm{~m} / \mathrm{sec}$, and a trajectory following error of $\pm 1.3 \mathrm{deg}$ for operating conditions.

\section{Bibliography}

[1] Park, J.W.; Lee, J.H.; Kim, J.W. (2003); Mathematical Model of Shock Absorber for Performance Prediction of Automobile, J. Korean society of marine engineers, ISSN 1226-9549, 27(4): $467-478$.

[2] Nunome, H. et al (2007); A Valid Shock Absorbency Test For Artificial Turf, J. of Biomechanics, ISSN 0021-9290, 40: 740.

[3] Choo, Y.G. (2013); A Study on Designing Autonomous Parking Assistance using Fuzzy Controller, J. of the Korean Society of Manufacturing Technology, ISSN 1598-6721, 12(1): 70-76.

[4] Tsiotras P.; Diaz R.S. (2014); Real-Time Near-Optimal Feedback Control of Aggressive Vehicle Maneuvers, Optimization and optimal control in automotive systems, ISSN 0170-8643, 1(1): 109-130. 
[5] Hossain, M.; Nabors, D.T. (2005); Testing and Evaluation of Used Automobile Tires and Recycled Tire-Derived Materials for Low-Cost Crash Cushions, J. of Materials in civil engineering, ISSN 0899-1561, 17(1): 36-44.

[6] Chung, M.J.; OH, S.G. (2008); Development of Auto Measuring System for Shock Absorbing Performance Test of Artificial Turf Ground, Int. Conference on Control, Automation and Systems, Print ISBN 978-89-950038-9-3, IEEE: 847-850.

[7] Chung, M.J.; OH, S.G.; Kang, K.W. (2010); A Study on the Evaluation of Shock Absorbing Performance for Domestic Artificial Turf Ground, Int. Journal of Modern Physics, ISSN 0217-9792, 24(15-16): 2567-2572.

[8] Chung, M.J.; Park, H.B.; Choi, K.H. (2012); Mathematical Model Based Attitude Control of Inverted Pendulum Type Mobile Robot, Conference on Institute of Control, Robotics and Systems, ICROS: 147-150. 